\title{
Tuning strain of granular matter by basal assisted Couette shear
}

\author{
Yiqiu Zhao ${ }^{1, \star}$, Jonathan Barés ${ }^{1,2, \star \star}$, Hu Zheng ${ }^{1,3, \star \star \star}$, and Robert Behringer ${ }^{1, \star \star \star \star}$ \\ ${ }^{1}$ Department of Physics, Duke University, Durham, NC, 27708 \\ ${ }^{2}$ Laboratoire de Mécanique et Génie Civil, Université de Montpellier, Montpellier, France 34090 \\ ${ }^{3}$ School of Earth Science and Engineering, Hohai University, Nanjing Jiangsu, China 210098
}

\begin{abstract}
We present a novel Couette shear apparatus capable of generating programmable azimuthal strain inside 2D granular matter under Couette shear. The apparatus consists of 21 independently movable concentric rings and two boundary wheels with frictional racks. This makes it possible to quasistatically shear the granular matter not only from the boundaries but also from the bottom. We show that, by specifying the collective motion of wheels and rings, the apparatus successfully generates the desired strain profile inside the sample granular system, which is composed of about 2000 photoelastic disks. The motion and stress of each particle is captured by an imaging system utilizing reflective photoelasticimetry. This apparatus provides a novel method to investigate shear jamming properties of granular matter with different interior strain profiles and unlimited strain amplitudes.
\end{abstract}

\section{Introduction}

Granular matter can be jammed by shear. A jammed granular system can sustain finite stress without irreversible deformation [1]. If the packing fraction $\phi$ of a granular system is larger than a particular value $\phi_{S}$, the system can transition from an unjammed state to a shear jammed state after a finite amount of shear strain [1-5]. In simulations, the shear strain is usually defined as the affine deformation driving individual particles and can be easily tuned [2, 5]. However, in the experimental case the shear strain is usually given by the deformation of the confining boundaries $[1,3,6,7]$. This boundary strain, although a practical tool, is not always useful to describe the deformation inside the granular matter. The shear jamming transition is achieved by percolation of a force network inside the granular system under shear [1]. The evolution of this force network is strongly related to the interior strain of the granular system $[1,6]$. Therefore the analysis of a stress-strain relation based on boundary strain may not be able to probe the intrinsic constitutive relation of granular matter undergoing a shear jamming transition.

To understand the interior strain of the granular matter, we present a novel apparatus that can generate boundary Couette shear with programmable interior strain field. The interior strain of the granular system corresponds to the displacements of each particle. This displacement field, or strain field will be called in this paper the resultant profile. And the collective motion of boundaries and bottom rings used to apply shear will be called the driving profile.

\footnotetext{
^e-mail: yiqiu.zhao@duke.edu

$\star \star$ e-mail: jb@jonathan-bares.eu

$\star \star \star$ e-mail: hz64@duke.edu

$\star \star \star \star$ e-mail: bob@phy.duke.edu
}

In this work, we compare the resultant and driving profiles for three kinds of shear: (i) boundary Couette shear (ii) linear shear and (iii) power-law shear with exponents $n=0.5$ and $n=2$. We show that this novel Couette shear apparatus leads to resultant profiles keeping the features of the driving profiles. In particular, we show that a linear shear results in a homogeneous density field even for very large boundary shear strains $(\sim 500 \%)$. By extracting stress information from the photoelastic patterns, we track the evolution of force network and probe the shear jamming transition. This apparatus provides a novel method to investigate the role played by the interior quasistatic strain of granular matter during shear jamming transition.

\section{Experiment}

The granular system studied here consists of about 2000 frictional (friction coefficient: $\mu \sim 0.6$ ) bi-disperse photoelastic disks. The bigger ones have a radius $R b=$ $7.95 \mathrm{~mm}$, while the smaller ones have a radius $R s=$ $6.35 \mathrm{~mm}$. The size ratio is kept constant for all experiments: $N_{\text {big }} / N_{\text {small }}=1 / 3$. The particles have reflective bottoms (see fig. 1(b)), making it possible to view the photoelastic patterns from the top using reflective photoelasticimetry [8]. We use a cooling system to ensure the room temperature remains the same to get rid of any possible thermal deformation of the particles. The system consists of 12 cooling fans to exhaust hot air heated by step motors and one thermal meter to monitor the temperature (fig. 1(d)). The room temperature is kept at $70^{\circ} \mathrm{F}$, at which the particles are free from any residual stress.

The particles are placed randomly onto the Couette shear apparatus shown in fig. 1. This Couette shear appara- 


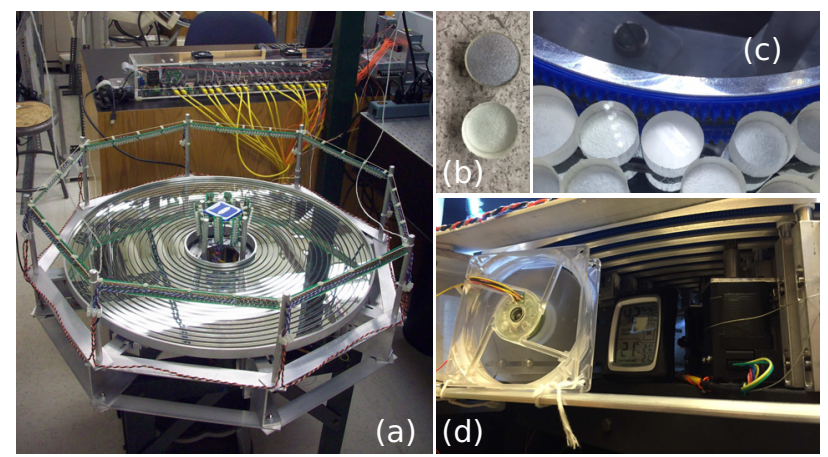

Figure 1: Experimental setup. (a) The Couette shear apparatus with outer boundary diameter $70.9 \mathrm{~cm}$ and inner boundary diameter $17.5 \mathrm{~cm}$. Concentric rings can be rotated independently. (b) The photoelastic particles. The upper particle is up side down, showing its painted reflective bottom. (c) Blue rack attached to to inner and outer boundaries to increase their roughness and apply boundary shear. (d) Cooling system. Left: fans to exhaust hot air. Right: Stepper motors controlling the motion of rings.

tus is made of two boundary wheels and 21 independently movable rings. Each ring has a width that is the same as the diameter of the smaller particles. As the ring rotates, it carries the particles on it. However, the friction between particle and ring $\left(\sim 4 \times 10^{-3} \mathrm{~N}\right)$ is smaller than any interparticle forces are visible as photoelastic patterns. The inner wheel and the inner-most ring of the apparatus are joined and the outer wheel moves with the out-most ring. Each ring but the inner-most one is controlled by a stepper motor beneath. Both boundary wheels have racks attached to apply boundary shear (see fig. 1(c)).

To apply shear to the system, both outer boundary and bottom rings move to drive the system. The shear is quasistatic, which means we shear the system step by step and take pictures between steps when the rings are stopped. For each step, the shear rate applied by the rings is in the order of $10^{-3} s^{-1}$. The motions of rings are controlled by 20 stepper motors. We call the relation between the number of steps a motor moves, $N(r)$, and the radial position of the ring controlled by the motor, $r$, the driving profile. The displacement of the ring is $N(r) \cdot d$, where $d=0.01431(9) \mathrm{mm}$ is the same for all rings. The driving strain applied by this ring is defined as $\epsilon(r)=N(r) \cdot d / r$ (see fig. 2 insert). The strain applied by the outer-most ring will be called the boundary strain.

The driving profile determines the collective motion of all the rings and boundaries. If $N(r)=c \cdot r$, we call this driving profile a linear profile, and the shear applied to the system in this way a linear shear. Similarly, if $N(r)=$ $c \cdot r^{n}$, we call the driving profile a power-law profile with exponent $n$, and this type of shear a power law shear with exponent $n$.

Each experimental run is composed of a series of quasi-static shear steps. Fig. 2 presents two driving profiles for one quasi-static steps. The driving profile is the same for all quasi-static steps of a given experimental run.
For every quasi-static steps, the motors move with different speed so that they start and stop at same time. The boundary shear displayed in fig. 2 means that only the outer-most ring and the outer boundary fixed to it are moving. This boundary shear can be regarded as a power-law shear with a very large exponent. As an example, the red curve demonstrates a fit with exponent $n=100$.

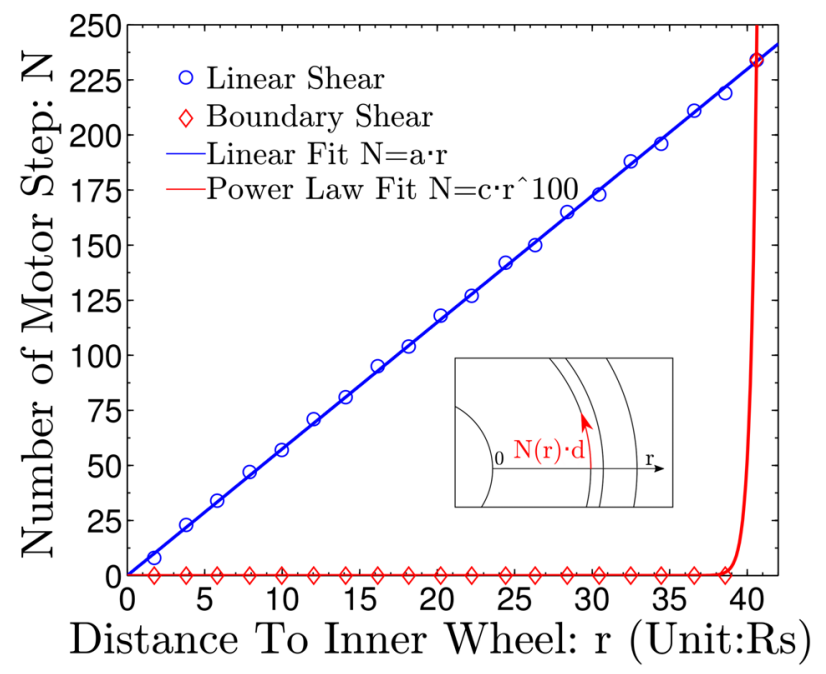

Figure 2: The driving profiles of linear and boundary shears. Main panel: The motor steps needed to generate linear and boundary shear for one quasi-static shear step. Inset: The azimuthal displacement of a ring at radial distance $r$ is $N(r) \cdot d$, where $d=0.01431(9) \mathrm{mm}$. The driving strain caused by this ring is $\epsilon(r)=N(r) \cdot d / r$.

The imaging system of the experiment is shown in fig. 3(a). A 20Mp CCD camera (Canon $\left.{ }^{\circledR} 70 \mathrm{D}\right)$ lays $3 \mathrm{~m}$ above the Couette shear apparatus to image the whole system. Between two consecutive quasi-static steps, the camera is triggered to take two pictures. The first one is taken under UV light (see fig. 1(a)). The particles are labeled by UV sensitive marks on their top, which permits us to find their position during picture post-processing. Such a picture is presented in fig. 3(b). Another picture is taken using reflective cross-polarized light coming from the green LED light surrounding the camera. This green light picture shows the photoelastic response of each particle (see fig. 3(c)).

\section{Result}

We call the displacements of particles resulting from the driving profile a resultant profile. The resultant profiles of the driving profiles shown in fig. 2 are displayed in fig. 4. In these experiments, the granular system under shear has a packing fraction of $\phi=0.7304$. The resultant profiles show the displacements of particles after 398 quasi-static shear steps, corresponding to $500 \%$ boundary strain. As shown in fig. 4, the azimuthal displacements of particles generated by linear shear are very well fitted by a straight line. However, this linear fit does not exactly goes 


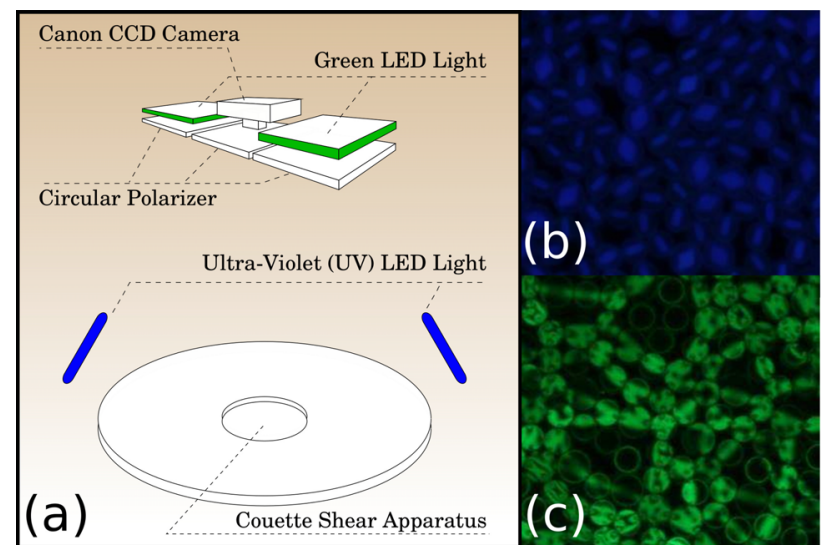

Figure 3: (a) Imaging system of the experiment. Particles placed on the shear apparatus can be luminated by two kinds of lights: (i) UV light from the UV LEDs surrounding the shear appratus and (ii) Green polarized light from the green LEDs surrounding the camera. (b) UV light image. Labels drawn on the particles using UV sensitive ink are luminated. Those labels are used to find centers and orientations of the particles. (c) Green light image. Reflective photoelasticity permits to observe stress inside particles. Green light is used because it is optimal for the circular polarizers we used.

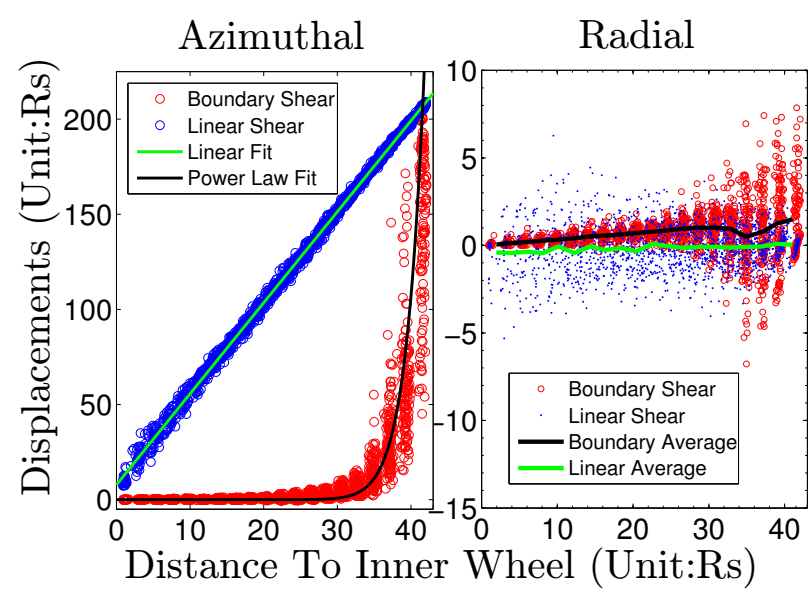

Figure 4: Resultant profiles of linear and boundary shears after $500 \%$ boundary strain. This example system has a packing fraction of $\phi=0.7304$ (see also fig. 2). Left panel shows the azimuthal displacements of particles v.s. their initial position. The linear fit slightly deviates from the origin. The power-law fit gives an exponent $n=16.93(34)$. The right panel shows the radial displacements of particles v.s. their initial positions. The positive direction is centripetal direction. Boundary shear results in a collective centripetal motion while linear shear does not.

through origin. This means there is a small global rotation of the whole system. The red circles in fig. 4 show the displacement of particles under boundary shear. For azimuthal displacements, the power law-fit gives an exponent $n=16.9(7)$, which is significantly less than the driv- ing profile $(n=100)$. But it keeps the feature of driven profile: the deformations are localized close to the outer boundary.
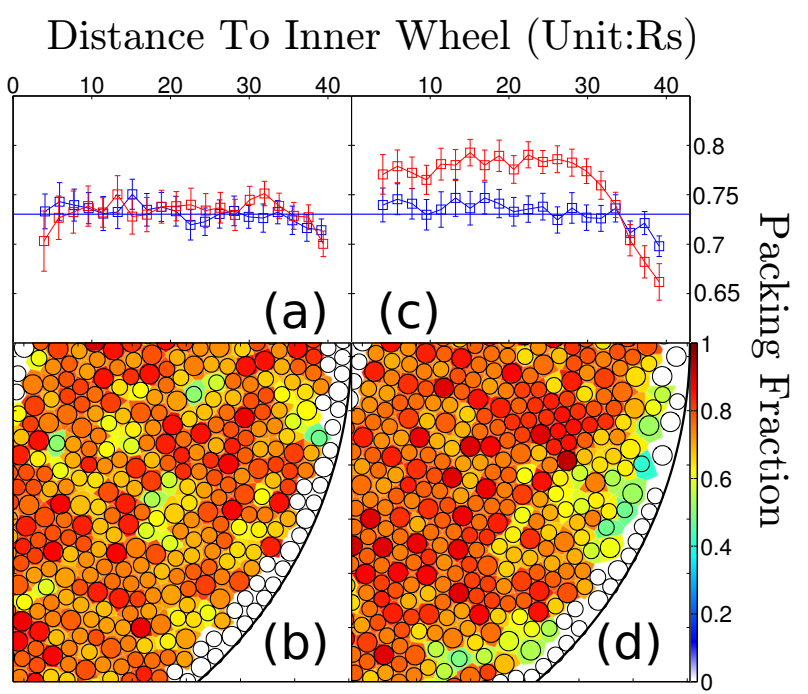

Figure 5: Shear-band study. (a) and (c) present the radial distribution of packing fractions before and after 500\% boundary strain for systems under linear shear (a) and boundary shear (c). The blue squares show the density distribution along the radial direction before shearing, while the red ones display the density after shearing. The blue lines indicate the system packing fraction $(\phi=0.7304)$. In (a) no low density area is created. But in (c) a localized low density band (shear band) is created after shear. (b) shows the local packing fraction computed from Voronoi cells after $500 \%$ linear shear. It is compared to the case where the system is sheared from boundaries in (d).

The right panel of fig. 4 shows the radial displacements of particles. The green curve shows the average displacements from the linear shear resultant profile. It fluctuates around zero which indicates there is no collective flow along the radial direction. However, as shown by the black curve, which is for boundary shear, the average radial displacement is non-zero and positive (towards ring center). This collective radial motion results in a shear band near the outer wheel. Fig. 5 shows the radial density (packing fraction) profile of the sample granular system before and after linear shear (a) and boundary shear (c). Blue squares show the density distribution before shear while red ones show the density distribution after $500 \%$ shear strain. The data points are averaged from the local packing fractions defined as particle area divided by the Voronoi area associated with the same particle. Error bars are at a 95\% confidence interval. We remark that after $500 \%$ shear strain, the boundary shear generates an obvious shear band (lower density area) with width around $10 \cdot R s$, while the system under linear shear remain homogeneous everywhere except for small fluctuations due to the intrinsic randomness of the granular systems.

We present the green light images for the sample system after $500 \%$ boundary strain under linear shear (see fig. 6(a)) and boundary shear (see fig. 6(b)). There are 


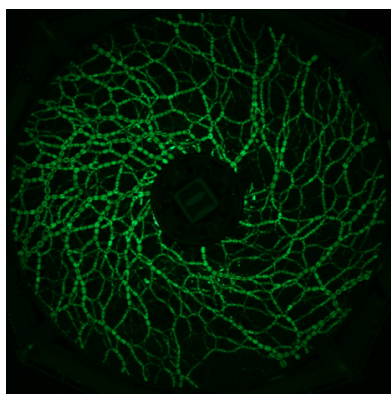

(a) Linear Shear

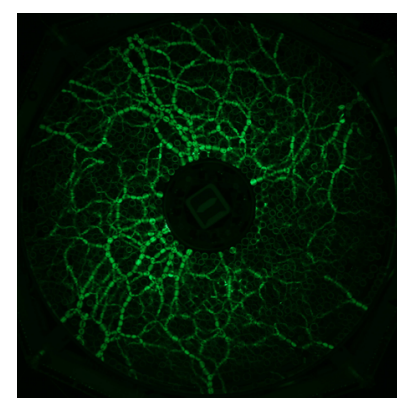

(b) Boundary Shear
Figure 6: The green light cross polarized image for system with $\phi=0.7304$ under linear (a) and boundary (b) driven profile after $500 \%$ boundary strain. The particle with brighter intensity bears larger force.

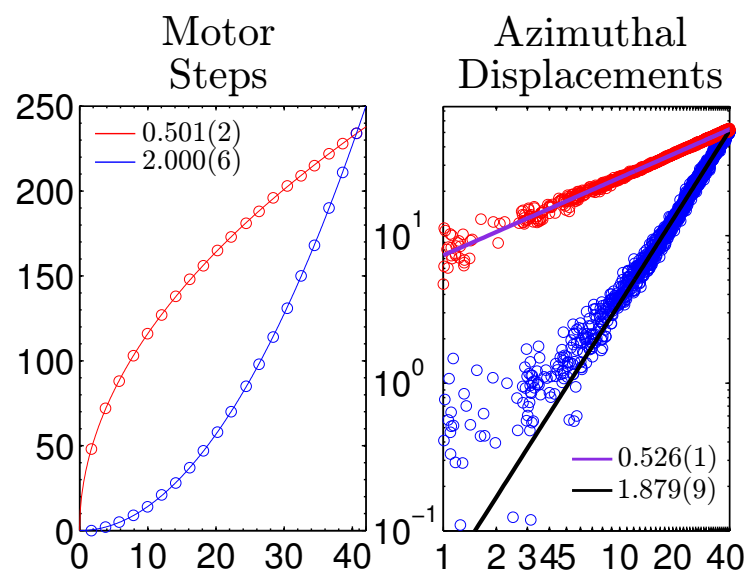

Distance To Inner Wheel (Unit:Rs)

Figure 7: Driving and resultant profiles for a granular system with packing fraction $\phi=0.7257$ after $125 \%$ boudnary shear strain. The left panel shows the number of motor steps for each ring needed to generate the power-law driving profiles. The right panel shows the resultant azimuthal displacements for individual particles (Unit: $R s$ ). The power-law fits show that driving profiles with exponents $0.501(2)$ and 2.000(6) generate resultant profiles with exponents $0.526(1)$ and $1.879(9)$ respectively.

two key observations from fig. 6: (i) the apparatus is able to generate resultant profiles retaining the features of the driving profiles even if the inter-particle forces are much larger than basal friction forces; (ii) by changing the interior strain we generate force networks with significantly different structures under the same boundary strain. Both observations confirm the ability of our apparatus to be used to study the relation between interior strain and shear jamming, and shear flows.

Besides linear and boundary shear, we can generate arbitrary programmable driving profiles. We present two more examples. Fig. 7 presents the driving and resultant profiles for a system with packing fraction $\phi=0.7257$ under power-law shear with exponents $n=0.5$ and $n=2$. The left panel shows the driving profile for an individual quasi-static step. And the right panel shows the resultant particle azimuthal displacement. Power-law fitting of the resultant profile shows that an $n=2$ driving profile results in an $n=1.879(9)$ resultant profile, and an $n=0.5$ driving profile results in an $n=0.526$ (1) resultant profile. Although the resultant exponents deviate slightly from the driving exponents, they are close enough to retain the main features of the driving profiles.

\section{Conclusion and outlook}

We have described a novel experimental system that can tune the interior deformation of granular systems under boundary Couette shear with basal assistance. The shear is applied by a novel Couette shear apparatus consisting of 21 independently movable rings and two boundary wheels. We present resultant profiles for three kinds of driving profiles: (i) boundary Couette shear, (ii) linear shear, and (iii) power-law shear with exponents 0.5 and 2. The resultant profiles keep the features of the driving profiles. In particular, we showed that under linear basal assisted shear, the granular system does not form shear bands even under very large boundary strains ( 500\%). This setup makes it feasible to investigate shear jamming properties of granular system by changing interior strains.

\section{Acknowledgement}

This work is supported by NSF-DMR1206351, DMS1248071, NASA NNX15AD38G.

\section{References}

[1] B.C. Dapeng Bi, Jie Zhang, R.P. Behringer, Nature 480, 355 (2011)

[2] N. Kumar, S. Luding, Granular Matter 18, 58 (2016)

[3] H. Zheng, J.A. Dijksman, R.P. Behringer, EPL (Europhysics Letters) 107, 34005 (2014)

[4] J. Zhang, T.S. Majmudar, M. Sperl, R.P. Behringer, Soft Matter 6, 2982 (2010)

[5] M. Baity-Jesi, C.P. Goodrich, A.J. Liu, S.R. Nagel, J.P. Sethna, ArXiv e-prints (2016), 1609.00280

[6] J. Ren, J.A. Dijksman, R.P. Behringer, Phys. Rev. Lett. 110, 018302 (2013)

[7] B. Utter, R.P. Behringer, Phys. Rev. E 69, 031308 (2004)

[8] J.G. Puckett, K.E. Daniels, Phys. Rev. Lett. 110, 058001 (2013) 